\title{
Cell-derived vesicles for delivery of cancer immunotherapy
}

\author{
Jialu Xu®, Chao Wang®* \\ Institute of Functional Nano \& Soft Materials (FUNSOM), Jiangsu Key Laboratory for Carbon-based Functional Materials and \\ Devices, Soochow University, Suzhou 215123, Jiangsu, China
}

*Correspondence: Chao Wang, Institute of Functional Nano \& Soft Materials (FUNSOM), Jiangsu Key Laboratory for Carbonbased Functional Materials and Devices, Soochow University, Suzhou 215123, Jiangsu, China. cwang@suda.edu.cn

Academic Editor: Haijun Yu, Chinese Academy of Sciences, China

Received: November 1, 2020 Accepted: November 27, 2020 Published: February 28, 2021

Cite this article: Xu J, Wang C. Cell-derived vesicles for delivery of cancer immunotherapy. Explor Med. 2021;2:39-59. https:// doi.org/10.37349/emed.2021.00031

\begin{abstract}
In recent years, cancer immunotherapy has received unprecedented attention due to the clinical achievements. The applications of biomedical engineering and materials science to cancer immunotherapy have solved the challenges caused by immunotherapy to a certain extent. Among them, cell-derived vesicles are natural biomaterials chosen as carriers or immune-engineering in view of their many unique advantages. This review will briefly introduce the recent applications of cell-derived vesicles for cancer immunotherapy.
\end{abstract}

\section{Keywords}

Cell-derived vesicles, immunotherapy, extracellular vesicles

\section{Introduction}

The immune system, as an essential component of the body, plays a significant role in prevention of occurrence and development of tumors [1-3]. Recently, cancer immunotherapy has received widespread attention due to its advantages in activating the body's immune system to fight cancer [4-8]. To date, cancer immunotherapy includes chimeric antigen receptor (CAR) T-cells, immune checkpoint blockade (ICB) therapies, and cancer vaccines are promise in cancer treatment [9-13]. Compared with traditional treatment approaches (surgery, chemotherapy, radiotherapy), cancer immunotherapy has relatively high efficiency and safety. Although immunotherapy has been clinically recognized, there are still a small number of patients (about 20\%) responding to it [14]. At the same time, immunotherapy causes numerous side effects, such as immune related adverse events $[15,16]$. The development of biomedical engineering and materials science provides new ideas for solving the challenges faced by cancer immunotherapy [17-21]. Among them, the application of biomaterials and nanotechnology in cancer immunotherapy has received extensive attention [22-24].

Unlike synthetic materials, cell-derived vesicles have become a research hotspot due to their inherent biocompatibility [25]. Cells are the basic structure and functional units that constitute organisms. The common structure of all cells includes cell membrane (consisting of phospholipid bilayer) and some organelles. Nowadays, a large number of studies have reported the applications of cell-derived vesicles in 
cancer immunotherapy and other diseases, including whole cell, and cell-derived membranous structures (e.g., exosomes and microvesicles) [26-31]. Whole cell refers to the direct application without any modification or transformation of cell. Cell-derived membranous structures are particles with double lipid membranes secreted or fabricated by cells, which cannot replicate like cells, but carry proteins, nucleic acids, lipids and other substances from mother cells. These applied cells can be red blood cells (RBCs), immune cells [e.g., macrophages, dendritic cells (DCs), natural killer cells (NK cells)], stem cells, cancer cells, bacteria, and so on (Figure 1). The application of cell-based vesicles are discussed in this review, and it is hoped that this review will help readers better understand the research progress of cell-based drug delivery systems.

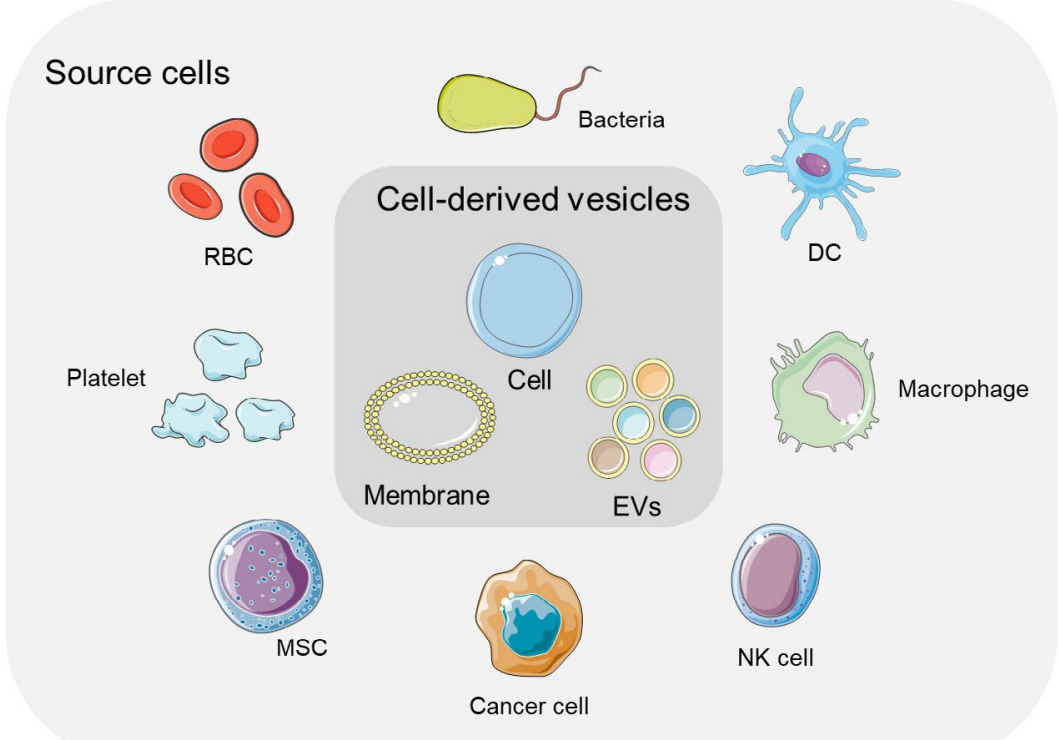

Figure 1. Schematic of cell-derived vesicles for drug delivery systems. EVs: extracellular vesicles; MSC: mesenchymal stem cells

\section{Cell derived vesicles}

RBC

RBCs, as the most abundant type of blood cells, are also named erythrocytes. Since mature RBCs do not have any nucleus, they can change shape at will when flowing in blood vessels, which is a feature that other synthetic materials do not possess [32]. They are utilized in drug delivery system due to their high loading capacity, low immunogenicity, good biocompatibility, and long circulation time in vivo [25, 33-35]. In addition to RBCs, RBC-membranes also can be exploited as drug carriers because of its semi-permeability, which can prevent the rapid removal of agents and achieve the effect of sustained release [36, 37]. In view of these advantages, Wang and his coworkers [36] designed numerous formulations based RBCs to deliver agents to prevent and treat some kinds of tumors. For example, they designed an RBC membrane loaded with tumor antigens and combined with anti-programmed death ligand-1 (anti-PD-L1) to form a cancer vaccine. Considering that RBCs are damaged or senescent, they will be eliminated by immune cells in the spleen, such as macrophages and DCs. For this reason, the nanovaccine can effectively target antigen-presenting cells (APCs) in the spleen and tumors. Related antigens are presented to $\mathrm{T}$ cells to activate $\mathrm{T}$ cells and cause immune response. This new type nanovaccine is fused by RBC membrane and the tumor cell membrane through ultrasound and membrane extrusion, namely nano-Ag@erythrosome. Tumor cell membranes provide tumor-associated antigens for activating T cells, while RBC membranes increase the circulation time of nanovaccine in the body and play a role in reducing toxicity and increasing efficacy. It was confirmed in B16F10 and 4T1 tumor models that the nanovaccine combined with antiPD-L1 could effectively inhibit tumor growth, which provided new ideas for cancer immunotherapy and the utilization of RBC membranes (Figure 2). Zhang and his coworkers [38] utilized RBC membranes to wrap perfluorohexane (PFC) and glucose oxidase (GOX) to construct an artificial NK cell (aNK) to mimic the role of NK cells in cancer immunotherapy. The novel artificial NK cells generated hydrogen peroxide $\left(\mathrm{H}_{2} \mathrm{O}_{2}\right)$ in tumor microenvironment, which directly killed tumor cells. In addition, the generated $\mathrm{H}_{2} \mathrm{O}_{2}$ 
recruited immune cells and activated macrophages to achieve the goal of suppressing tumor cells. At the same time, PFC improved the hypoxia of the tumor microenvironment and further activated the immune system to inhibit tumor growth (Figure 3). Although RBCs, as the abundant cells in the body, have their unique advantages, they do not have an advantage in the ability of targeting.

\section{A}
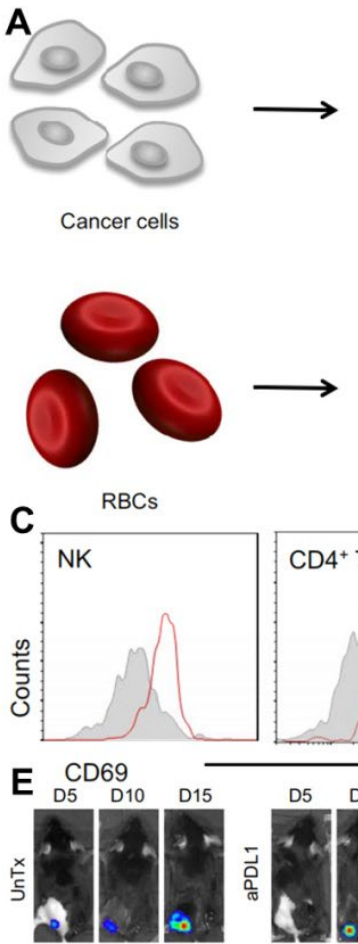

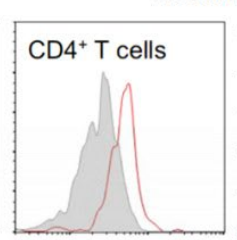

RBC Membran

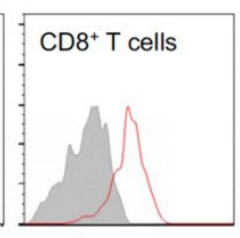

Cancer cell membrane
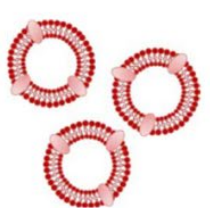

D5 $\quad$ D10 D15

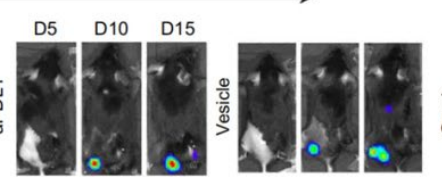

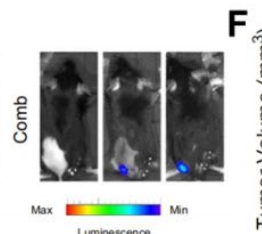

B

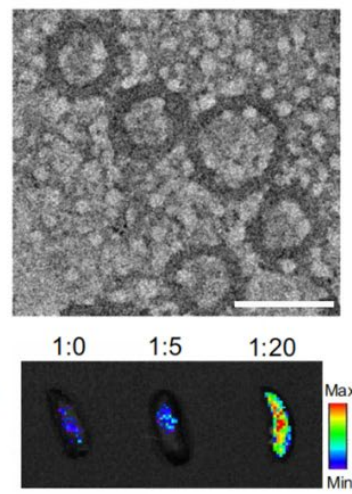

CD19+B cells

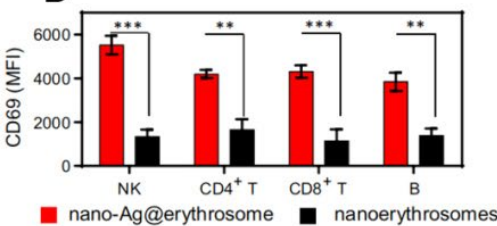

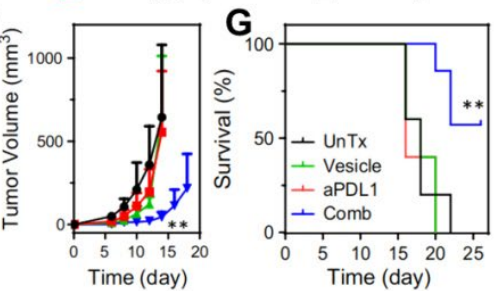

Figure 2. (A) The preparation of nanovaccine; (B) TEM imaging of nano-Ag@erythrosome and ex vivo imaging of spleen $1 \mathrm{~h}$ after intravenous injection at various ratios; (C) activation markers measured $24 \mathrm{~h}$ after intravenous injection in splenic immune cell subsets (red for treatment, gray for control); (D) corresponding quantification of MFI according to C; (E) in vivo bioluminescence imaging of the distant B16F10-Luc tumors after different treatments as indicated; (F) the tumor growth curves of B16F10; (G) survival curves for the treated and control mice

Note. Adapted from "Red blood cell-derived nanoerythrosome for antigen delivery with enhanced cancer immunotherapy," by Han X, Shen S, Fan Q, Chen G, Archibong E, Dotti G, et al. Sci Adv. 2019;5:eaaw6870 (https://advances.sciencemag.org/content/5/10/ eaaw6870). CC BY-NC.

A

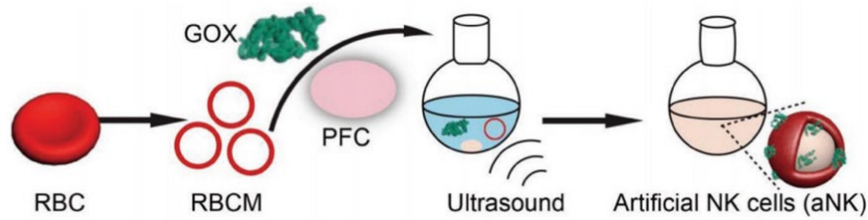

B

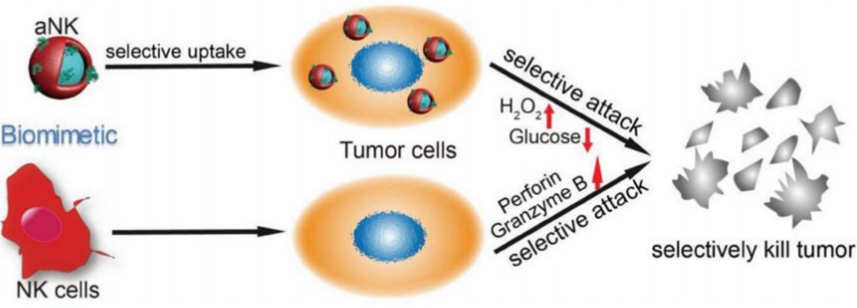

C

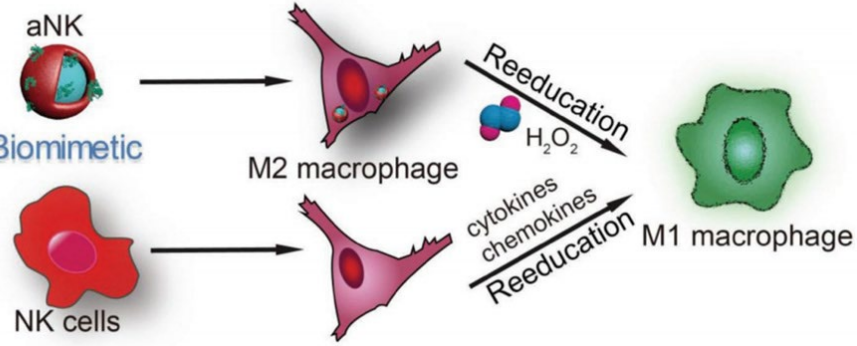


Figure 3. (A) The preparation of aNK; (B) the evaluation of the function of aNK and NK cells to kill tumor selectively; (C) aNK and NK cells induce the polarization of macrophages

Note. Adapted from "Artificial natural killer cells for specific tumor inhibition and renegade macrophage re-education," by Zou MZ, Liu WL, Gao F, Bai XF, Chen HS, Zeng X, et al. Adv Mater. 2019;31:e1904495 (https://onlinelibrary.wiley.com/doi/epdf/10.1002/ adma.201904495). CC BY-NC.

\section{Immune cell}

Immune cells are an important part of immune system and can help the body fight infections and other diseases. Immune cells are developed from stem cells in the bone marrow, including lymphocytes (B cells and T cells), APCs (macrophages and DCs), neutrophils, NK cells [39-43]. In vivo and in vitro studies have shown that immune cells can be targeted and activated to secrete different pro-inflammatory cytokines to induce immune responses. In addition, immune cells are widely used in drug delivery systems with their own unique advantages, which reduce the difficulties faced by other therapies to a certain extent. Here, we will introduce in detail the application of extracellular vesicles (EVs) derived from immune cells in tumor immunotherapy [44].

EVs are released by cells with a membranous structure that can participate in cell-to-cell communication [30, 45-47]. EVs can be roughly divided into three types: micro-vesicles, apoptotic bodies, and exosomes $[48,49]$. Among them, the diameter of exosomes is about $30-150 \mathrm{~nm}$, which can transfer RNA, functional proteins, lipids and metabolites to recipient cells in the body, and actively participate in the communication between the host and pathogen cells and organs [50-52]. Most cells can secrete and ingest exosomes. Exosomes secreted by immune cells can mediate innate immunity and adaptive immunity, and regulate cancer progression and metastasis. Therefore, exosomes derived from immune cells are applied as drugs or carriers to play a role in cancer immunotherapy [53-55].

\section{B cell and T cell}

Lymphocytes are a type of white blood cell in the immune system [56]. T cells and B cells are the main components of the adaptive immune responses. T cells participate in cellular immunity, and B cells are responsible for humoral immunity. The function of them is to recognize specific "non-self" antigens. Once recognizing foreign objects, they will produce specific responses to eliminate foreign objects. B cells fight pathogens by producing a large number of antibodies, and $\mathrm{T}$ cells cause immune responses by producing cytokines [57]. Regulatory $\mathrm{T}$ cells (Treg) are a subgroup of $\mathrm{T}$ cells that maintain tolerance to themselves and limit other immune responses at the same time. A key target for Treg to exert immunomodulatory function is DCs [58]. The miRNAs exist on the surface of Treg-derived EVs can be taken up by DCs, which activate DCs to produce pro-inflammatory to inhibit tumor growth [59]. Yang et al. [60] found that EVs secreted by $\mathrm{T}$ cells could promote the secretion of IgG from B cells and T cells utilized in this work were firstly activated by pyruvate kinase muscle isozyme 2 (PKM2). Next, when the EVs were endocytosed by B cells, the programming and activation of mitochondria, and the production of IgG would be inhibited. Lu et al. [61] purified $\mathrm{CD} 4^{+} \mathrm{T}$ cell EVs from $\mathrm{CD} 4^{+} \mathrm{T}$ cells activated in vitro. After immunization with Treg vaccine, the mice treated with $\mathrm{CD} 4^{+} \mathrm{T}$ cell EVs showed a strong humoral immune response. At the same time, they found that EVs played an important role in the response of B cells in vitro, and could significantly promote the activation, proliferation and produce antibody of $\mathrm{B}$ cells. Therefore, EVs derived from $\mathrm{CD} 4^{+} \mathrm{T}$ cells could be applied as a new immunomodulatory to promote antigen-specific humoral immune responses. Zhang et al. [62] found that CD19+ EVs extracted from B cells highly expressed CD39 and CD73, which could hydrolyze ATP released by tumor cells after chemotherapy into adenosine, thereby inhibiting $\mathrm{CD} 8^{+} \mathrm{T}$ cell response and reducing tumor treatment effects.

\section{APC}

APCs can be divided into two categories, professional APCs and non-professional APCs. Professional APCs express major histocompatibility complex (MHC) II, costimulatory molecules and pattern recognition receptors, including macrophages and DCs. Macrophages play a vital role in innate immunity, which are present in all organs [63]. Macrophages can drive the accumulation of carriers in chronically inflammatory tumor tissues due to their natural inflammation-directed chemotaxis [64-66]. Zhou and his colleagues [67] 
designed tumor-associated macrophage (TAM) derived EVs to treat epithelial ovarian cancer (EOC). Because TAM-EVs expressed miR-29a-3p and miR-21-5p, when TAM-EVs interacted with CD4 ${ }^{+} \mathrm{T}$ cells, they directly inhibited signal transducer and activator of transcription 3 (STAT3) and regulated Treg/Th17 cells. The results of this study revealed that EVs mediated the interaction between TAMs and T cells, which had a certain impact on the progress and metastasis of EOC, which provided a new idea for the treatment of EOC. Rayamajhi et al. [68] fused EVs derived from macrophages with synthetic liposomes to form hybrid exosomes (HE), which expressed CD81, CD63 and CD9 on the surface. The water-soluble Doxorubicin (DOX) was loaded into HE, and DOX could be released in the acidic microenvironment of the tumor, thereby inhibiting tumor growth. DCs can induce humoral and cellular immunity by presenting antigens to $\mathrm{T}$ cells $[69,70]$. Recent researches have confirmed that DC-derived EVs express MHC class I and MHC class II molecules, which have the ability to combine with proteins to active T cells. So, DCs-derived EVs can be utilized to induce anti-tumor immune responses [71-73]. Different subtypes of EVs were derived from human primary DCs, and the effects of EVs on primary CD4+ $\mathrm{T}$ cells were studied in vitro. The results showed that EVs secreted by immature DC could effectively activate T cells, promote Th2 cells to secrete cytokines interleukin-4 (IL-4), interleukin-5 (IL-5), and interleukin-13 (IL-13), and promote Th1 cells to secrete interferon- $\gamma$ (IFN- $\gamma$ ). For EVs secreted by mature DCs, IFN- $\gamma$ was mainly secreted, which was a potential strategy for anti-tumor immune response [74]. Another study showed that EVs derived from mouse bone marrow dendritic cells (BMDCs) could induce higher levels of $\mathrm{CD}^{+} \mathrm{T}$ cells to inhibit the growth of melanoma. At the same time, EVs-derived from BMDCs induced powerful antibody-specific immunity and provided long-term immunity [75].

\section{Neutrophil}

Neutrophils are important immune cells in tumor environment, which have dual functions of pro-tumors and anti-tumors [76]. Researches have shown that neutrophils themselves have the ability to penetrate the blood-brain barrier and target brain tumors[77]. Surgical removal of tumor will cause local inflammation, releasing inflammatory cytokines, such as IL-8 and tumor necrosis factor (TNF)- $\alpha$, thereby activating the migration of neutrophils to the inflammatory environment of brain [78]. In view of above advantages, Xue and co-workers [79] utilized neutrophils to encapsulate pentatonix (PTX)-loaded liposomes, which could migrate to the inflammatory environment after intravenous injection. After reaching the tumor site, PTX was released from liposomes into tumor, thereby inducing cytotoxicity and inhibiting tumor recurrence after surgery. Gao and his coworkers [80] designed a new approach, nitrogen cavitation, to extract vesicles from neutrophils and encapsulated piceatannol, which could significantly reduce lipopolysaccharide (LPS)induced acute lung injury and sepsis, thereby decreasing inflammation and improving survival rate. This phenomenon was mainly due to the inhibition of the NF- $\kappa B$ pathway in endothelial cells after the vesicle was loaded with piceatannol. This research mainly proposed a new strategy for the extraction of EVs, and could be applied to the preparation of other types of EVs.

\section{NK cell}

NK cells were first discovered in 1975 and are a kind of innate effector lymphocytes, which are different from T cells and B cells $[81,82]$. In vitro studies have shown that NK cells can kill a variety of tumor cells, and they are particularly effective in removing metastatic cells, however, they are not effective in removing large solid tumors [83-86]. NK cells can recognize and kill abnormal cells restricted by MHC or not pre-sensitized, and are considered to be the most effective immune cell subset for monitoring and eliminating diseased cells in the body $[87,88]$. At the same time, NK cells can secrete some inflammatory cytokines to regulate the immune response [89]. In view of these, the treatment based on NK cells has been reported recently [90, 91]. Zhu et al. [92] applied density gradient ultracentrifugation to extract EVs from NK cells. NK cells they applied in this experiment were treated with IL-15. With the help of IL-15, the yield of EVs increased, and it could target tumor site effectively and had stronger anti-tumor ability to glioblastoma. Wang and co-workers [93] reported on a novel cocktail therapy that combined excess NK cell-derived EVs with nanoparticles for targeted tumor therapy. In vivo experiments showed that the new EVs loaded with nanoparticles had the ability to efficiently target and treat neuroblastoma with good biocompatibility. Zhang and co-workers [94] designed 
an artificial engineered NK cells combined with photothermal therapy to improve the efficiency of treatment of solid tumors. The novel CONASHs were constructed by self-assembly of metal ions and organic dyes. After being modified with polyetherimide (PEI) and DNAzyme, CONASHs had good photothermal conversion both in vivo and in vitro. In addition, through the strategy of metabolic polysaccharide biosynthesis, NK cells were modified with hepatocellular carcinoma HCC-specific targeting aptamers (TLS11a), which enabled NK cells to have active targeting capabilities. Combining adoptive therapy with artificial NK cells and photothermal therapy (PTT) had achieved excellent and significantly improved anti-tumor efficiency in vivo (Figure 4). Therefore, they emphasized that this programmable treatment strategy has the potential as an effective tumor treatment [94].

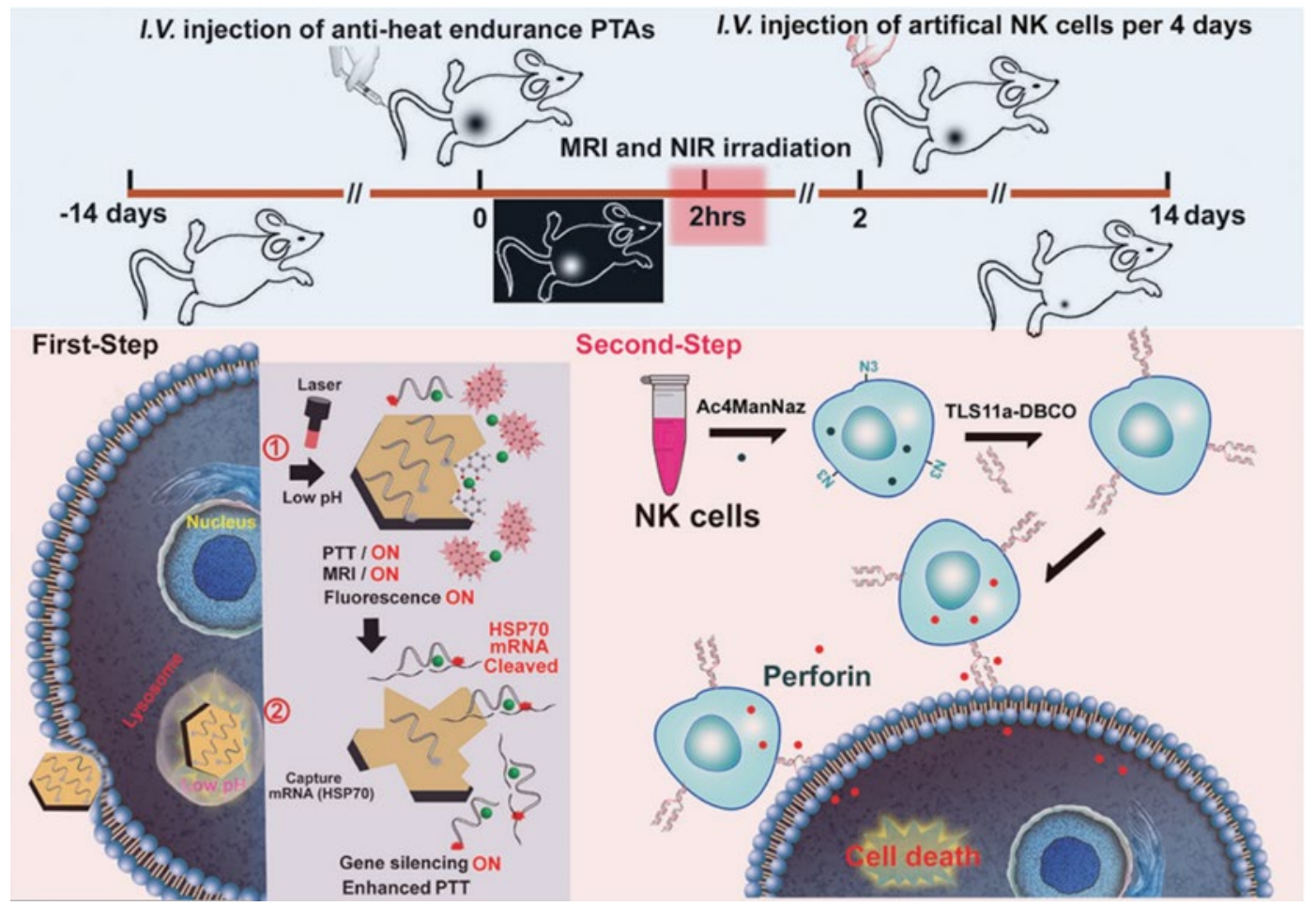

Figure 4. Schematic illustration of artificial engineered NK cells combined with antiheat endurance strategy for improving the therapeutic efficiency of PTT. A simple strategy to artificially engineer NK cells by aptamers has been developed, and it has been further combined with antiheat endurance of DNAzyme to act as powerful strategy for immuno-enhancing the therapeutic

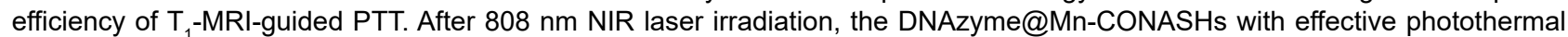
conversion ability could damage tumors and subsequently release $\mathrm{Mn}^{2+}$ by tumor acidiccleaving the coordination bonds between $\mathrm{Mn}^{2+}$ and THAQ, serving as cofactors to DNAzyme for HSP70 gene silencing to overcome heat resistance of tumor cells during PTT. Finally, the TLS11a aptamer decorated artificial NK cells could actively target and effectively kill the residual unkilled or resistant tumor cells after PTT to improve the completeness of tumor removal

Note. Reprinted from "Artificial engineered natural killer cells combined with antiheat endurance as a powerful strategy for enhancing photothermal-immunotherapy efficiency of solid tumors," by Zhang D, Zheng Y, Lin Z, Lan S, Zhang X, Zheng A, et al. Small. 2019;15:e1902636 (https://onlinelibrary-wiley-com.virtual.anu.edu.au/doi/10.1002/smll.201902636). Copyright WILEYVCH Verlag GmbH \& Co. KGaA, Weinheim.

\section{Platelet}

Other immune cells, such as platelets, can also be applied in antitumor responses. Platelets are nonnucleated cells in the blood circulation. They can activate and attach to the injured area to prevent bleeding. Recently, platelets and platelet-derived EVs have been applied in cancer immunotherapy [31, 95-97]. As early as 2017, Wang et al. [98] proposed using platelets as a carrier to modify anti-PD-L1 on the surface to prevent recurrence and metastasis after surgical removal of tumors. It was verified on mice with B16F10 and 4T1 tumor models, and the results showed that the platform could effectively release the anti-PD-L1 antibody, and significantly prolonged the survival rate of mice after surgery, reducing the rate of cancer regeneration (Figure 5). In another work, Han and co-workers [96] designed a platelet-based platform to deliver anti-PD-L1 checkpoint antibodies to inhibit tumor recurrence. Considering that platelets have good inflammation targeting ability, they hoped to apply anti-PD-L1 platelet monoclonal antibody to efficiently transport anti-PD-L1 antibody to the area of residual tumor ablation, thereby effectively inhibiting local 
tumor recurrence. In 4T1 tumor mice, they proved that antibody-conjugated platelets could effectively target tumors that were not completely ablated by thermal ablation. At the same time, they found that when platelets were activated, they could promote the release of anti-PD-L1 and secret many pro-inflammatory cytokines. The results of the study confirmed that the platelet platform they designed promoted the delivery of immune checkpoint antibodies to tumor residues and significantly prevented tumor recurrence after ablation. In addition, this platelet-based drug delivery strategy could be extended to targeted drug delivery after other local treatments.
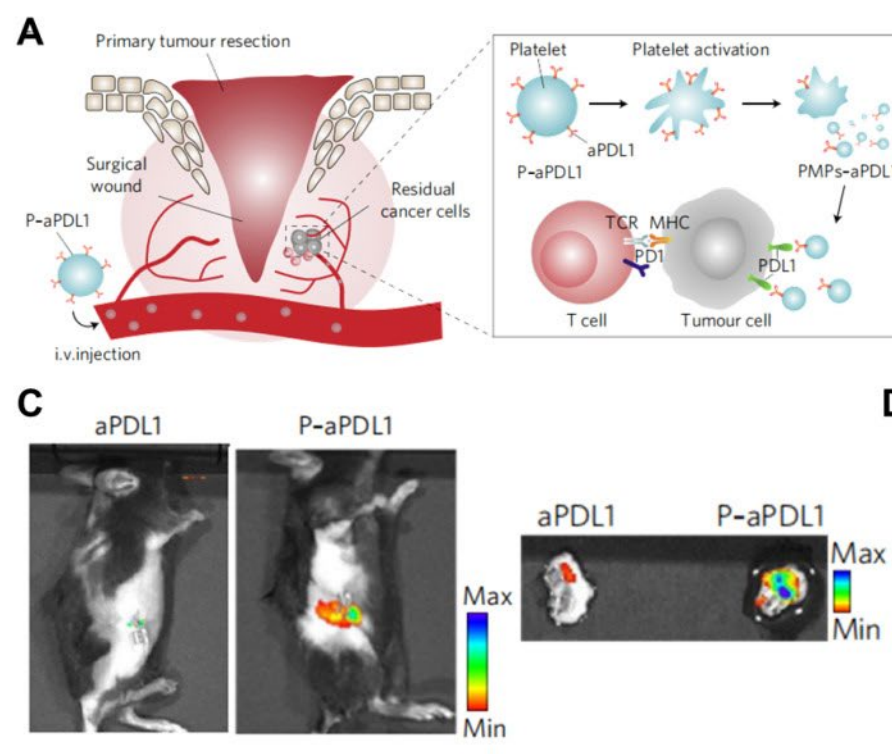
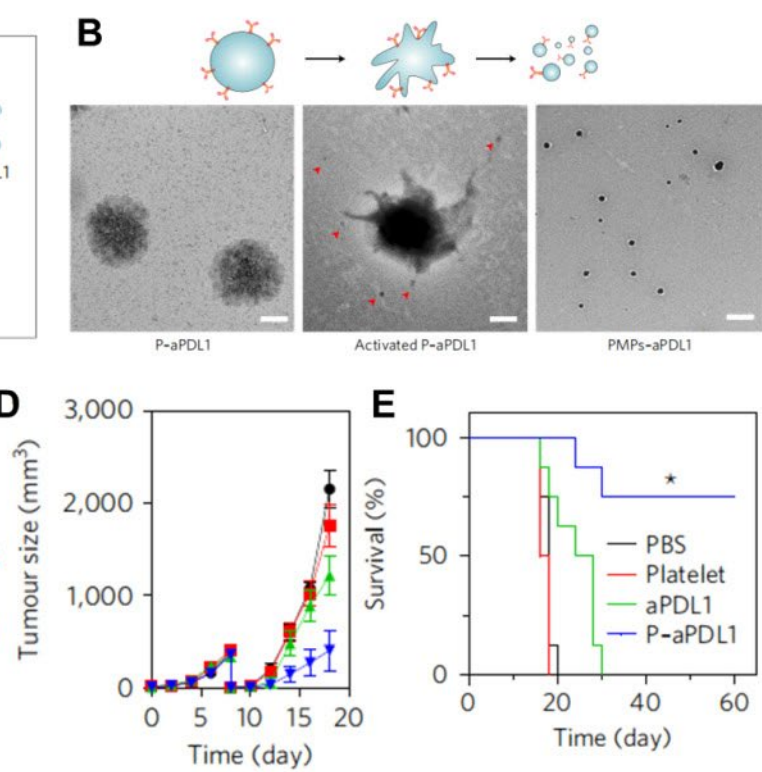

E

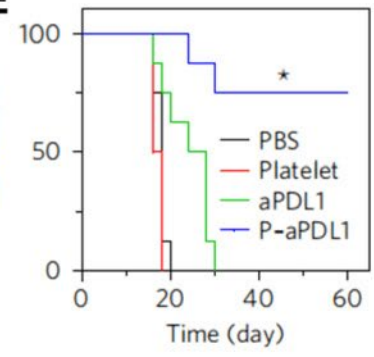

Figure 5. (A) Schematic illustration of the delivery of anti-PDL1 to the primary tumor resection site by platelets; (B) TEM imaging of different formulations; $(C)$ fluorescence imaging of wounds with residual tumors after i.v. injection of platelet anti-PDL1 or free anti-PDL1; (D\&E) the tumor growth curves and survival curves of different groups of B16F10 tumor model after administrating different formulations

Note. Adapted from "In situ activation of platelets with checkpoint inhibitors for post-surgical cancer immunotherapy," by Wang C, Sun W, Ye Y, Hu Q, Bomba HN, Gu Z, Nat Bio Engineer, 2017;1:0011 (https://www. nature.com/articles/s41551-016-0011). CC BY-NC.

\section{Stem cell}

Mesenchymal stem cells (MSCs) are a heterogeneous cell population with self-renewal and continuous differentiation [99, 100]. So far, MSCs have been widely utilized in clinical trials, and achievements have been made in the treatment of autoimmune diseases and inflammatory diseases [101-105]. To date, chimeric antigen receptor T-Cell (CAR-T) therapy has achieved good results in the treatment of leukemia and other diseases, however, its applications in solid tumors have been greatly restricted. Abken and co-workers [106] took advantages of the ability of MSCs to actively migrate to the tumor site and modified MSCs with IL-17 and IL-12. The modified MSCs could interact with CAR-T cells to release IFN- $\gamma$ and TNF- $\alpha$, thus improving the activation and increase of CAR-T cells. They were the first to apply MSCs to regulate the expression of cytokines in tumor microenvironment to improve the efficacy of CAR-T therapy in solid tumors [107].

EVs secreted by MSCs have been shown to have immunomodulatory functions, capable of delivering agents loaded by the parent cells to recipient cells without tumorigenicity or variability [108, 109]. EVs derived from MSCs exert immune tolerance in the innate and adaptive immune response. MSC-EVs can inhibit the activation and proliferation of $\mathrm{T}$ cells as they can improve the function of Treg and the production of IL-22 [109]. Researches have confirmed the function of MSC-EVs on T cells. Similarly, MSC-EVs also have inhibitory effects on B cell proliferation, differentiation and immunoglobulin secretion. Taking into account innate immune cells, MSC-EVs can induce the regulatory phenotype of DC expressing IL-10 and inhibit the co-stimulatory molecules of monocytes [110, 111]. Bruno et al. [112] found that EVs derived from human bone marrow MSCs inhibited the cycle progression of cancer cells, including HepG2 liver cancer, and induced necrosis of cancer cells. Intratumor injection of MSC-EVs significantly inhibited the growth of tumors, indicating that MSC-derived EVs played an important role in the anti-tumor immune response. In addition, 
MSC-EVs can be applied as carriers to deliver agents to tumor environment. EVs are safer compare to other delivery systems. Pascucci et al. [113] encapsulated paclitaxel into MSC-EVs, and when targeted to human pancreatic cancer, antitumor drugs were released from MSC-EVs, and the therapeutic effect was dosedependent, and the tumor suppression rate could reach $50 \%$.

\section{Cancer cell}

Cancer cells can proliferate indefinitely and form solid tumors or abnormal cells that flood the blood $[114,115]$. Due to the uniqueness of specific proteins and ligands on the surface of cancer cells, after being used in the modification of nanoparticles, it can not only target homotypic tumors, but also improve the treatment efficiency, which has received widespread attention [116, 117]. So far, Zhang and co-workers [118] have explored numerous formulations based cell membrane to induce antitumor responses. In 2019, they reported a biomimetic nanoparticle loaded with photosensitizer and hydrophobic drug (HCPT) into 4T1 tumor cell membrane. The loaded photosensitizer indocyanine green (ICG) not only realized photothermal treatment, but also improved the curative effect of hydrophobic drugs. It was found in breast cancer tumor models that the biomimetic nanoparticles effectively inhibited tumor growth and possessed good biological safety (Figure 6).

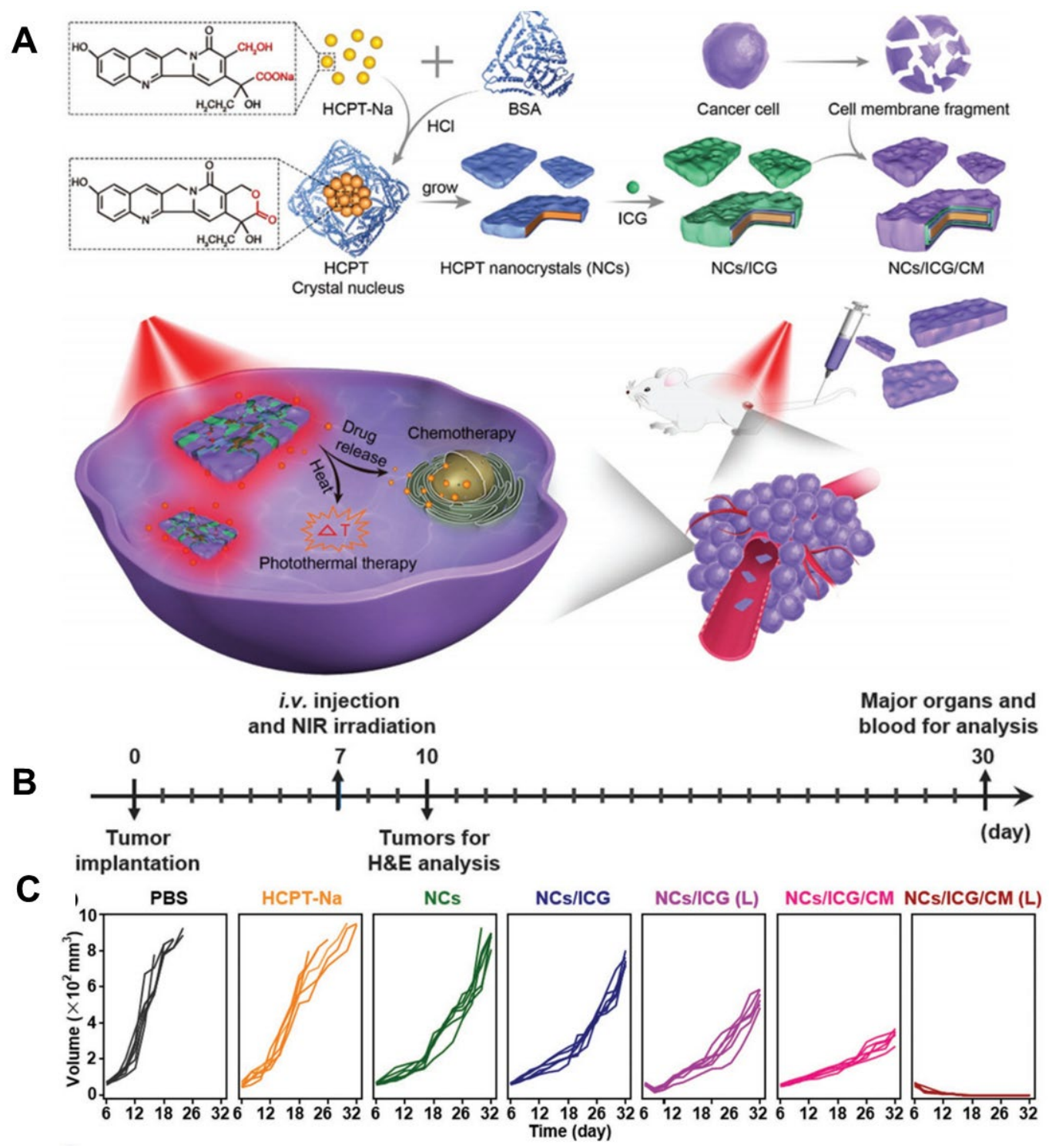

Figure 6. (A) Schematic illustrating cancer cell membrane-coated HCPT with photosensitizer for the treatment of orchestration; (B) scheme of the treatment of orchestration with different formulations; (C) the tumor growth curves of different groups of 4T1 tumor model after administrating vaccines

Note. Adapted from "Cell membrane camouflaged hydrophobic drug nanoflake sandwiched with photosensitizer for orchestration of chemo-photothermal combination therapy," by Zhang L, Zhang X, Lu G, Li F, Bao W, Song C, et al. Small. 2019;15:1902648 (https://onlinelibrary.wiley.com/doi/abs/10.1002/smll.201805544). CC BY-NC. 
EVs derived from colorectal cancer cell have the ability to interfere with intracellular signals to inhibit the proliferation of T cells [119]. EVs were extracted from highly metastatic pancreatic cancer cell lines by ultracentrifugation. When incubated with NK cells, it was found that the levels of CD71 and CD98 on the surface of NK decreased, and the ability to take up glucose was affected [120]. Fleming and co-workers [121] isolated EVs (Ret-EVs) from Ret melanoma cell lines, which could induce programmed death ligand-1 (PD-L1) upregulation in bone marrow-derived mouse immunological memory cell (IMC) and immortalized bone marrow suppressor cell lines. This is mediated by Toll-like receptor (TLR) signaling pathways and heat shock proteins. The results showed that EVs induced PD-L1 expression provided a promising target for tumor immunotherapy by mediating the transformation of normal bone marrow cells into Myeloid-derived suppressor cells (MDSC) by TLR signaling pathways. Another study designed a DC-targeted tumor vaccine modified with EVs derived from melanoma (ApoEVs). Before apoptosis induced by bortezomib, it activated high mannose glycans with DC-specific C-type lectin-like cell-surface receptor (DC-SIGN, CD209) binding on tumor cells, resulting in high expression of high mannose on ApoEVs, which helped to enhance the uptake of DCs through DC-SIGN, thereby activating antigen-specific $\mathrm{CD} 8^{+} \mathrm{T}$ cells. These data indicated that using glycosylated ApoEVs as carriers can selectively deliver tumor-derived antigens to DCs in vivo to initiate tumor-specific CD8 ${ }^{+} \mathrm{T}$ cell responses [122].

In conclusion, vesicles derived from cancer cells contain specific antigens, which can effectively target homotypic tumors. At the same time, they can also be processed into nanovaccine, which can promote the maturation of DCs and use antigen to extract. APCs could present the antigen on the surface of cancer cells to $\mathrm{T}$ cells, thereby activating $\mathrm{T}$ cells, causing a series of immune responses, and providing new ideas and methods for cancer immunotherapy. Nowadays, cancer cell-derived vesicles have achieved good results in different tumor models. However, in view of the clinical situation, it is still difficult to truly achieve clinical transformation.

\section{Bacteria}

Bacteria are single-celled organisms different from plants and animals. In appearance, they are usually several microns long and live in tens of thousands of groups. Structurally, they do not have a nucleus, so they belong to prokaryotes. They are usually divided into two categories, namely, Gram-positive bacteria and Gram-negative bacteria, and the biggest difference between them is the presence of cell wall. Bacterial-based cancer therapy can be traced back to 1891 . Since then, considering that bacteria have good phagocytosis and can be a pathogen-associated molecular pattern (PAMP), they trigger a series of immune responses after interacting with pattern recognition receptors (PRR). They can be carriers encapsulating agents to target APCs. A large number of studies have shown that bacteria can selectively colonize to the hypoxic areas of tumors, thereby inhibiting tumor growth [123-125]. Leventhal et al. [126] applied synthetic biology technology to develop the Escherichia coli strain SYNB1891, which had the ability to target APCs in tumors, activating the stimulator of interferon genes (STING) pathway, and trigger complementary innate immune responses. SYNB1891 produced high levels of cell differentiation agent (CDA) in mouse and human APCs, and at the same time, it induced the production of IFN by phagocytosis. In mouse tumor models, SYNB1891 produced effective anti-tumor immunity and immune memory. What is exciting is that the first phase of clinical trials for intratumoral injection of SYNB1891 for advanced or metastatic tumors has begun. Danino and co-workers [127] designed a probiotic system using a stable lysis release mechanism to control the production and intratumoral release of PD-L1 and cytotoxic T-lymphocyte-associated protein 4 (CTLA-4). Compared with similar clinically relevant antibodies, a single injection of this probiotic-based drug delivery system showed a stronger therapeutic response, resulting in tumor regression in a syngeneic mouse model, and the number of activated $\mathrm{T}$ cells was relatively increased. Finally, they combined with granulocytemacrophage colony stimulating factor (GM-CSF) in a syngeneic mouse model with poor immunogenicity demonstrated its highly effective therapeutic effect. Together, these results indicated that a novel engineered probiotic system connected synthetic biology and immunology to improve ICB therapies.

To date, tumor targeted microorganisms have become cancer specific drug delivery vehicles or therapeutic agents, such as Bacillus Calmette-Guerin, anaerobic bacteria, and oncolytic viruses [128]. Due to 
the hypoxia, immunosuppression and unique biochemical microenvironment in solid tumors, these bacteria can be selectively colonized in tumor tissues [129-131]. It is understood that certain types of bacteria can deliver near infrared (NIR) absorbers to tumors for photothermal ablation. However, without the addition of other therapeutic agents or nanoparticles, phototherapy or photo-immunotherapy using bacteria alone has not yet been achieved. Recently, Yi and co-workers [132] proposed a photo-immunotherapy applying bacteria entirely. Their research results showed that after intravenous injection of attenuated Salmonella, it would proliferate in various types of solid tumors, and the inflammation caused by the bacterial nanovaccine would trigger thrombosis in the infected tumor by destroying tumor blood vessels, which was conducive to effective tumor ablation. In addition, photothermal therapy triggered by bacteria could also induce an effective anti-tumor immune response. Specifically, they first determined the location of attenuated Salmonella in mice. They found that $72 \mathrm{~h}$ after the injection, the bacteria had gradually been eliminated from the major organs (such as the heart, liver, spleen, kidney, and lungs). Next, they went on to investigate the effect of attenuated Salmonella on tumors. Results indicated that the colonization of attenuated Salmonella in tumors can trigger tumor-specific thrombosis by destroying tumor blood vessels. In addition, with the help of antiCTLA-4 checkpoint blocking therapy, bacteria-based photothermal therapy can effectively inhibit the growth of distant tumors and spontaneous tumor metastasis. And at the same time, they could provide long-term immune memory effects, thereby protecting mice from the recurrence of tumors.

\section{Virus}

Virus-like particles (VLPs) are a spherical or tubular protein nanostructure formed by self-assembly of virus capsid protein (CP). They have a structure similar to natural virus capsid, without genome and infection. VLPs have shown unique research and application value in many fields such as vaccine, targeted drug delivery, biomedical imaging and tissue engineering [133]. So far, varieties of virus-derived vesicles have been combined with immune checkpoint therapy, and achieved exciting results [134]. Virus can not only dissolve cells to activate the immune response, but also change the tumor microenvironment [135]. Kerstetter-Fogle and co-workers [136] reported a novel VLPs named Cowpea mosaic virus like particles (CPMV), which derived from plant cowpea. They applied CPMV to treating glioma, and the study proved that administration of CPMV immunotherapy in situ could effectively recruit innate immune cells and adaptive immune cells into the brain parenchyma, while also reducing the number of immunosuppressive cells, leading to regression of intracranial glioma. CPMV nanovaccine provided an effective and safe local immunotherapy for intracranial glioma. Zhang and co-workers [137] implanted viral antigens into the cell membrane through genetic engineering, and then formed uniform spherical virus mimic nanovesicles (VMVs) under the action of the active agent, which were similar to natural viruses in size, shape, and immunogenicity. First, the viral antigen genes were encapsulated into the lentiviral plant liked virus (PLV) vector. After selecting puromycin, human emborynic kidney (HEK) 293T cells expressing viral antigens on the cell membrane were obtained. Then, it was located in the endoplasmic reticulum through the recognition of the signal peptide. The sorting signal peptide guided the antigen protein into the plasma membrane with the help of transport vesicles. Vesicles carry antigen proteins fused with cell membranes, anchoring viral antigens on the cell surface. Finally, HEK 293T cells expressing viral antigens were incubated with sodium deoxycholate to produce major membrane vesicles. In order to verify the immune protection of VMV-hyaluronic acid (HA) in vivo, they injected 50 times the lethal dose of murine adapted influenza virus into mice intranasally. After treatment, they found that VMV-HA induced a protective immune response in the body. And in terms of immunogenicity, VMV vaccine could be matched with purified HA subunit vaccine (Figure 7). The VMVs they designed can be used as a vaccine delivery vector, using a variety of probes to surface functionalize VMVs, which can be used for targeted delivery. Given that VMVs can be inserted into large proteins in their natural form, VMVs nanotechnology can be applied to vaccine design for a variety of enveloped viruses, including vaccines with pandemic potential.

Oncolytic virus is considered to be anti-tumor drug because it can selectively replicate and kill cancer cells. Oncolytic virus not only can be retained in normal tissues, but also spread to tumors to induce immune response [138, 139]. Jiang et al. [140] designed the oncolytic virus Delta-24-RGDOX with the immune- 
stimulatory molecule OX40 ligand (OX40L), which could induce immunogenic cell death and recruit cells to the tumor site. When combined with anti-PD-L1 in a mouse model of glioma, it showed that the combination therapy could inhibit tumor growth and significantly improved the survival rate of mice. Their research showed that combining oncolytic viruses and immune checkpoint inhibitors induced effective autologous tumor in situ immunization, resulting in effective and durable tumor-specific therapeutic effects.

A

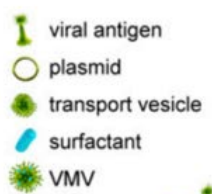

\section{G}

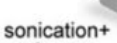

sonication+

*.* VMV
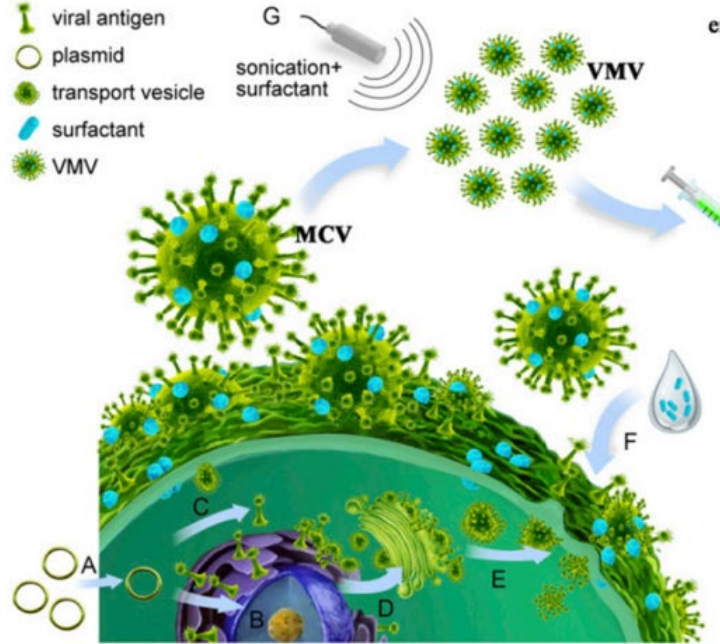

C
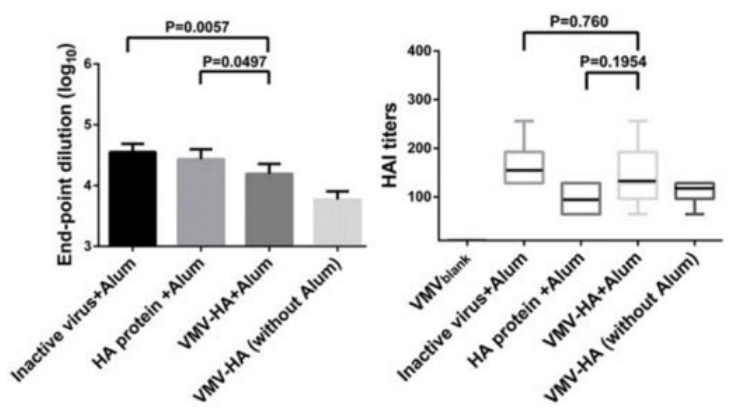
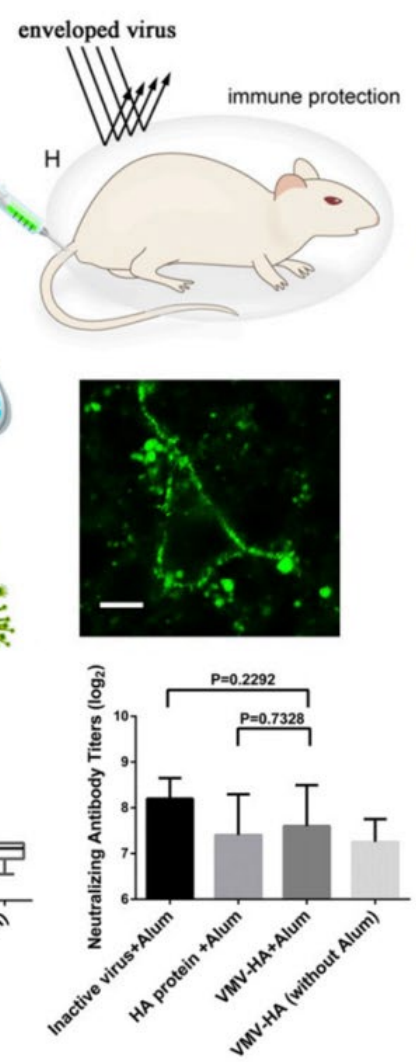

B

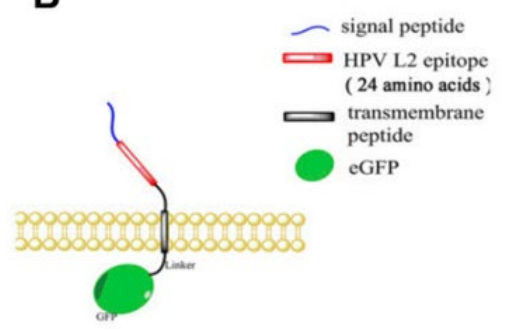

D
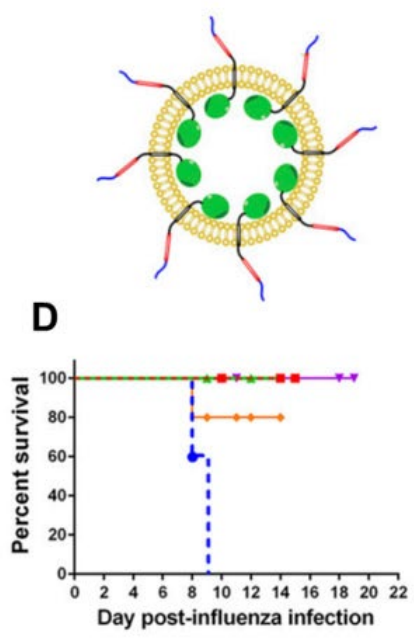

Figure 7. (A) Schematic revealing the preparation of virus mimic nanovesicles (VMVs) for encapsulating anti-tumor epitopes or enveloped-virus glycoprotein to VMV surface; (B) design and schematic representation of VMV displaying Sig-L2-eGFP; (C\&D) evaluation of the immunogenicity of VMV-HA

Note. Adapted from "Virus-mimetic nanovesicles as a versatile antigen-delivery system," by Zhang P, Chen Y, Zeng Y, Shen C, Li R, Guo Z, et al. PNAS, 2015;112:E6129-38 (https://www.pnas.org/content/112/45/E6129.long). CC BY-NC.

\section{Fungus}

In our living environment, there are microorganisms, compared with bacteria, fungi are more complicated organisms. Among these microorganisms, fungi are most similar to animals in their structure. There are two main types of fungi, the first one is environmental fungi, including yeast and mold, which do not generally cause infection in most healthy people; another is commensals fungi, which live on and in us and generally do not hurt us. In view of recent bacterial therapy and virus therapy [141-143], researches on fungi are attracting more and more attentions. At present, relatively more studies have been made on cell walls derived from Saccharomyces Cerevisiae. On the cell wall of the outer layer of yeast, there are a large number of polysaccharides and proteins, such as $\beta$-glucan, chitosan, mannoprotein, and so on [144]. $\beta$-glucan, as the most abundant ingredient, can bind to Dectin-1 receptors on immune cells to cause a series of immune responses including phagocytosis [145-147]. Cell wall derived from yeast can be utilized as an adjuvant to activate APCs, and next induce immune responses. Apart from these, the cell wall can be applied to deliver numerous agents, such as antigens, drugs. Yang [148] and his coworkers used an inverse micro-emulsion method to prepare antigen-loaded $\beta$-glucan particles (GPs). GPs can effectively activate DCs and produce cytokines, thereby delivering antigens to $\mathrm{T}$ cells and inducing humoral and cellular immune responses. At the same time, GPs can achieve the effects of preventing and treating mouse T lymphoma cells (EG7-OVA) tumors. In another work, they encapsulated an alum colloid inside GPs [149]. This particle owned a uniform size of 2-4 $\mu \mathrm{m}$. Similar to the previous particles, it can efficiently target APCs and lymph nodes, activate DCs and 
produce corresponding pro-inflammatory cytokines, thereby generating an anti-tumor immune response. In conclusion, the cell wall derived from yeast is a good adjuvant to induce cellular and humoral immune response to antitumor.

Vesicles derived from virus, bacteria, and fungus can lose the ability to reproduce after processing, their application may be restricted in terms of safety and the selection of raw material.

\section{Plant cells}

Plants are one of the main forms of life, and they are a kind of eukaryotic organisms. Similar to mammals, plants also have the structure of cell. The typical characteristics of plant cells are a large central vacuole, chloroplast, and stable cell wall. Nowadays, although a large number of studies have reported that mammalian cell-derived EVs have made important achievements in tumors and other diseases $[28,150,151]$, mammalian cell-derived EVs still have certain shortcomings, such as high product cost. In order to overcome the problems of mammalian cell-derived EVs, researchers began to explore plantderived EVs such as fruits and vegetables $[152,153]$. The results showed that these vesicles not only have the function of treating related diseases, but also can be used as a carrier to deliver a variety of agents. The novel delivery system provides new ideas and methods for the treatment of tumors and diseases. Currently, the differential centrifugation method has been utilized to extract EVs from plants. The whole process can be roughly summarized as follows: (1) juicing the washed plants; (2) removing large particles and fibers at a low speed; (3) removing large debris and organelles at a medium speed; (4) the precipitate obtained at high speed is EVs. Zhuang [154] and co-workers designed novel particles from ginger to protect against alcohol-induced liver damage by orally administration. EVs derived from plant cells have their unique advantages. They have no great toxicity or immunogenicity and can be loaded with multiple agents. In addition, the most important point is that its production cost is lower. In view of the above advantages, from the current point of view, plant-derived EVs are a research hotspot at present, and its application to tumor immunotherapy is a new direction.

\section{Conclusion}

Cell-derived vesicles have attracted widespread attention due to their inherent biocompatibility in recent years (Table 1) [155]. As the basic unit of organism, cell is mainly composed of outer cell membrane and inner organelle. The cell-based drug delivery systems can be roughly divided into three parts. First, whole cells own all components and properties of cells, hence the vesicles have the ability of high loading capacity, low immunogenicity, good biocompatibility and long circulation time. For example, RBCs can be loaded with ovalbumin (OVA), and be co-delivered with adjuvant poly (I:C) to mice, they can be effectively taken up by macrophages and DCs in the spleen, thereby activating antitumor immune response [156]. As produced above, platelets can be used to deliver anti-PD-L1 to the surgical site, thereby inhibiting tumor metastasis and recurrence. Second, use the cell membrane as a carrier to deliver agents. Because the cell membrane is a structure shared by all cells, any cell membrane can be used as a carrier. There are tumor-associated antigens on the surface of tumor cell membranes, which can be targeted to homotypic tumors, which provides the possibility for personalized treatment of tumors. Third, the EVs secreted by cells are also the current research hotspot. EVs and liposomes are similar in structure, which possess phospholipid bilayer. There are also a variety of proteins, mRNA, and miRNA in EVs, which can be targeted to specific tissues and organs. In general, as an emerging carrier, cell-derived vesicles can overcome the challenges of immunotherapy to a certain extent, and provide the possibility for the clinical transformation of cancer immunotherapy. For now, most cell-derived vesicles are still in the stage of the exploration of animals, and not many are actually applied for patients. Therefore, continuous efforts and explorations are required to achieve true clinical transformation. In addition, the combination therapy of vesicles from different cell sources may also be a new field and opportunity. 
Table 1. Summary of the properties and advantages of cell-derived vesicles

\begin{tabular}{llll}
\hline Platform & Properties & Advantages & Reference \\
\hline Whole cell & $\begin{array}{l}\text { Own the components and properties of cell, such as cell } \\
\text { membrane, organelles, etc. }\end{array}$ & $\begin{array}{l}\text { High loading capacity; } \\
\text { Low immunogenicity; }\end{array}$ \\
& & Good biocompatibility; \\
& & Long circulation time. & Wide variety of sources; $[30,45-47]$ \\
$\begin{array}{l}\text { Cell-derived } \\
\text { membranous structures }\end{array}$ & Participate in cell-to-cell communication; & Cannot replicate like cells; \\
& $\begin{array}{l}\text { Carry proteins, nucleic acids, lipids and other substances } \\
\text { from mother cells. }\end{array}$ & & \\
\hline
\end{tabular}

\section{Abbreviations}

aNK: artificial NK cell

anti-PD-L1: anti-programmed death ligand-1

APCs: antigen-presenting cells

CAR: chimeric antigen receptor

CAR-T: chimeric antigen receptor T-cell

CPMV: Cowpea mosaic virus

DC: dendritic cell

EOC: epithelial ovarian cancer

EVs: extracellular vesicles

GPs: glucan particles

$\mathrm{H}_{2} \mathrm{O}_{2}$ : hydrogen peroxide

IFN- $\gamma$ : interferon- $\gamma$

MHC: major histocompatibility complex

MSCs: mesenchymal stem cells

NK: natural killer

PD-L1: programmed death ligand-1

RBC: red blood cell

RBCs: red blood cells

TAM: tumor-associated macrophage

Treg: regulatory $\mathrm{T}$ cells

VLPs: virus-like particles

VMV: nanovesicles

VMVs: virus mimic nanovesicles

\section{Declarations}

\section{Author contributions}

Both authors contributed to the writing of the manuscript, and edited the manuscript at all stages. JX was responsible for collecting and writing the manuscript. CW was responsible for writing, reviewing and editing the manuscript.

\section{Conflicts of interest}

The authors declare that they have no conflicts of interest. 


\section{Ethical approval}

Not applicable.

\section{Consent to participate}

Not applicable.

\section{Consent to publication}

Not applicable.

\section{Availability of data and materials}

Not applicable.

\section{Funding}

This work was supported by grants from startup supports of Soochow University and the Program for Jiangsu Specially-Appointed Professors to CW. This work was also supported by National Natural Science Foundation of China (No. 31900988, 32022043), the Natural Science Foundation of Jiangsu Province (No. SBK2019040088) and Jiangsu Province Six Talent Peaks Project (No. SWYY-110). This work was partly supported by Collaborative Innovation Center of Suzhou Nano Science \& Technology, the Priority Academic Program Development of Jiangsu Higher Education Institutions (PAPD), the 111 Project. The funders had a role in the design and preparation of the manuscript.

\section{Copyright}

(c) The Author(s) 2021.

\section{References}

1. Carr EJ, Dooley J, Garcia-Perez JE, Lagou V, Lee JC, Wouters C, et al. The cellular composition of the human immune system is shaped by age and cohabitation. Nat Immunol. 2016;17:461-8.

2. Irvine DJ. Materializing the future of vaccines and immunotherapy. Nat Rev Mater. 2016;1:15008.

3. Goldberg MS. Improving cancer immunotherapy through nanotechnology. Nat Rev Cancer. 2019;19:587-602.

4. Klevorn LE, Teague RM. Adapting cancer immunotherapy models for the real world. Trends Immunol. 2016;37:354-63.

5. Khalil DN, Smith EL, Brentjens RJ, Wolchok JD. The future of cancer treatment: immunomodulation, CARs and combination immunotherapy. Nat Rev Clin Oncol. 2016;13:273-90.

6. Zhang P, Zhai Y, Cai Y, Zhao Y, Li Y. Nanomedicine-based immunotherapy for the treatment of cancer metastasis. Adv Mater. 2019;31:e1904156.

7. Topalian SL, Taube JM, Pardoll DM. Neoadjuvant checkpoint blockade for cancer immunotherapy. Science. 2020;367:eaax0182.

8. Yu W, He X, Yang Z, Yang X, Xiao W, Liu R, et al. Sequentially responsive biomimetic nanoparticles with optimal size in combination with checkpoint blockade for cascade synergetic treatment of breast cancer and lung metastasis. Biomaterials. 2019;217:119309.

9. Curran KJ, Silverman LB, Kobos R, Kernan NA, Margossian SP, Park JH, et al. Abstract IA07: chimeric antigen receptor T cells for cancer immunotherapy. AACR; 2016.76 Suppl:IA07.

10. Topalian SL, Drake CG, Pardoll DM. Immune checkpoint blockade: a common denominator approach to cancer therapy. Cancer Cell. 2015;27:450-61.

11. Fesnak AD, June CH, Levine BL. Engineered T cells: the promise and challenges of cancer immunotherapy. Nat Rev Cancer. 2016;16:566-81. 
12. Miliotou AN, Papadopoulou LC. CAR T-cell therapy: a new era in cancer immunotherapy. Curr Pharm Biotechnol. 2018;19:5-18.

13. Irvine DJ, Dane EL. Enhancing cancer immunotherapy with nanomedicine. Nat Rev Immunol. 2020;20:321-34.

14. Sharma P, Hu-Lieskovan S, Wargo JA, Ribas AJC. Primary, adaptive, and acquired resistance to cancer immunotherapy. Cell. 2017;168:707-23.

15. Luoma AM, Suo S, Williams HL, Sharova T, Sullivan K, Manos M, et al. Molecular pathways of colon inflammation induced by cancer immunotherapy. Cell. 2020;182:655-71.e22.

16. Singh B, Schwartz JA, Sandrock C, Bellemore SM, Nikoopour E. Modulation of autoimmune diseases by interleukin (IL)-17 producing regulatory T helper (Th17) cells. Indian J Med Res. 2013;138:591-4.

17. Shields CW, Wang LL, Evans MA, Mitragotri S. Materials for immunotherapy.Adv Mater. 2020;32:e1901633.

18. Christofi T, Baritaki S, Falzone L, Libra M, Zaravinos A. Current perspectives in cancer immunotherapy. Cancers (Basel). 2019;11:1472.

19. Riley RS, June CH, Langer R, Mitchell MJ. Delivery technologies for cancer immunotherapy. Nat Rev Drug Discov. 2019;18:175-96.

20. Nam J, Son S, Park KS, Zou W, Shea LD, Moon JJ. Cancer nanomedicine for combination cancer immunotherapy. Nat Rev Mater. 2019;4:398-414.

21. Xu X, Li T, Shen S, Wang J, Abdou P, Gu Z, et al. Advances in engineering cells for cancer immunotherapy. Theranostics. 2019;9:7889-905.

22. Fan $\mathrm{Q}$, Chen $\mathrm{Z}$, Wang C, Liu Z. Toward biomaterials for enhancing immune checkpoint blockade therapy. Adv Funct Mater. 2018;28:1802540.

23. Cai L, Xu J, Yang Z, Tong R, Dong Z, Wang C, et al. Engineered biomaterials for cancer immunotherapy. MedComm. 2020;1:35-46.

24. Fan Q Wang C. Engineering nanobiomaterials for cancer immunotherapy. Sci Sin Vitae. 2020;50.

25. Wang C, Wen D, Gu Z. Cellular bioparticulates with therapeutics for cancer immunotherapy. Bioconjug Chem. 2018;29:702-8.

26. Tian H, Li W. Dendritic cell-derived exosomes for cancer immunotherapy: hope and challenges. Ann Transl Med. 2017;5:221.

27. Shi $X$, Cheng $Q$ Hou T, Han M, Smbatyan G, Lang JE, et al. Genetically engineered cell-derived nanoparticles for targeted breast cancer immunotherapy. Mol Ther. 2020;28:536-47.

28. Yan W, Jiang S. Immune cell-derived exosomes in the cancer-immunity cycle. Trends Cancer. 2020;6:506-17.

29. Di Pace AL, Tumino N, Besi F, Alicata C, Conti LA, Munari E, et al. Characterization of human NK cellderived exosomes: role of DNAM1 receptor in exosome-mediated cytotoxicity against tumor. Cancers (Basel). 2020;12:661.

30. Kato T, Fahrmann JF, Hanash SM, Vykoukal J. Extracellular vesicles mediate B cell immune response and are a potential target for cancer therapy. Cells. 2020;9:1518.

31. Ma Q, Fan Q, Xu J, Bai J, Han X, Dong Z, et al. Calming cytokine storm in pneumonia by targeted delivery of TPCA-1 using platelet-derived extracellular vesicles. Matter. 2020;3:287-301.

32. Hamidi M, Tajerzadeh H. Carrier erythrocytes: an overview. Drug Deliv. 2003;10:9-20.

33. Gao M, Liang C, Song X, Chen Q, Jin Q, Wang C, et al. Erythrocyte-membrane-enveloped perfluorocarbon as nanoscale artificial red blood cells to relieve tumor hypoxia and enhance cancer radiotherapy. Adv Mater. 2017; [Epub ahead of print].

34. Yan J, Yu J, Wang C, Gu Z. Red blood cells for drug delivery. Small Methods. 2017;1. 
35. Pishesha N, Bilate AM, Wibowo MC, Huang NJ, Li Z, Deshycka R, et al. Engineered erythrocytes covalently linked to antigenic peptides can protect against autoimmune disease. Proc Natl Acad Sci U S A. 2017;114:3157-62.

36. Han X, Shen S, Fan Q, Chen G, Archibong E, Dotti G, et al. Red blood cell-derived nanoerythrosome for antigen delivery with enhanced cancer immunotherapy. 2019;5:eaaw6870.

37. Han X, Wang C, Liu Z. Red blood cells as smart delivery systems. Bioconjug Chem. 2018;29:852-60.

38. Zou MZ, Liu WL, Gao F, Bai XF, Chen HS, Zeng X, et al. Artificial natural killer cells for specific tumor inhibition and renegade macrophage re-education. Adv Mater. 2019;31:e1904495.

39. Schafer C, Ascui G, Ribeiro CH, Lopez M, Prados-Rosales R, Gonzalez PA, et al. Innate immune cells for immunotherapy of autoimmune and cancer disorders. Int Rev Immunol. 2017;36:315-37.

40. Wang H, Mooney DJ. Biomaterial-assisted targeted modulation of immune cells in cancer treatment. Nat Mater. 2018;17:761-72.

41. Lee DA. Cellular therapy: adoptive immunotherapy with expanded natural killer cells. Immunol Rev. 2019;290:85-99.

42. Shimasaki N, Jain A, Campana D. NKcells for cancer immunotherapy. Nat Rev Drug Discov. 2020;19:200-18.

43. Wilgenhof S, Corthals J, Heirman C, van Baren N, Lucas S, Kvistborg P, et al. Phase II study of autologous monocyte-derived mRNA electroporated dendritic cells (TriMixDC-MEL) plus ipilimumab in patients with pretreated advanced melanoma. J Clin Oncol. 2016;34:1330-8.

44. Robbins PD, Morelli AE. Regulation of immune responses by extracellular vesicles. Nat Rev Immunol. 2014;14:195-208.

45. Tetta C, Ghigo E, Silengo L, Deregibus MC, Camussi G. Extracellular vesicles as an emerging mechanism of cell-to-cell communication. Endocrine. 2013;44:11-9.

46. Becker A, Thakur BK, Weiss JM, Kim HS, Peinado H, Lyden D. Extracellular vesicles in cancer: cell-to-cell mediators of metastasis. Cancer Cell. 2016;30:836-48.

47. Mathieu M, Martin-Jaular L, Lavieu G, Thery C. Specificities of secretion and uptake of exosomes and other extracellular vesicles for cell-to-cell communication. Nat Cell Biol. 2019;21:9-17.

48. Raposo G, Stoorvogel W. Extracellular vesicles: exosomes, microvesicles, and friends. J Cell Biol. 2013;200:373-83.

49. Susa F, Limongi T, Dumontel B, Vighetto V, Cauda V. Engineered extracellular vesicles as a reliable tool in cancer nanomedicine. Cancers (Basel). 2019;11:1979.

50. Pasini L, Ulivi P. Extracellular vesicles in non-small-cell lung cancer: functional role and involvement in resistance to targeted treatment and immunotherapy. Cancers (Basel). 2019;12:40.

51. Maacha S, Bhat AA, Jimenez L, Raza A, Haris M, Uddin S, et al. Extracellular vesicles-mediated intercellular communication: roles in the tumor microenvironment and anti-cancer drug resistance. Mol Cancer. 2019;18:55.

52. Czystowska-Kuzmicz M, Sosnowska A, Nowis D, Ramji K, Szajnik M, Chlebowska-Tuz J, et al. Small extracellular vesicles containing arginase- 1 suppress T-cell responses and promote tumor growth in ovarian carcinoma. Nat Commun. 2019;10:3000.

53. Xu R, Rai A, Chen M, Suwakulsiri W, Greening DW, Simpson RJ. Extracellular vesicles in cancer-implications for future improvements in cancer care. Nat Rev Clin Oncol. 2018;15:617-38.

54. Katsuda T, Kosaka N, Ochiya T. The roles of extracellular vesicles in cancer biology: toward the development of novel cancer biomarkers. Proteomics. 2014;14:412-25.

55. Tominaga N, Yoshioka Y, Ochiya T. A novel platform for cancer therapy using extracellular vesicles. Adv Drug Deliv Rev. 2015;95:50-5.

56. Al-Shura AN. Advanced hematology in integrated cardiovascular chinese medicine. Amsterdam: elsevier; 2020. 
57. Paolillo M, Schinelli S. Integrins and exosomes, a dangerous liaison in cancer progression. Cancers (Basel). 2017;9:95.

58. Yan J, Liu B, Shi Y, Qi H. Class II MHC-independent suppressive adhesion of dendritic cells by regulatory T cells in vivo. J Exp Med. 2017;214:319-26.

59. Tung SL, Boardman DA, Sen M, Letizia M, Peng $Q$, Cianci N, et al. Regulatory T cell-derived extracellular vesicles modify dendritic cell function. Sci Rep. 2018;8:6065.

60. Yang J, Dang G, Lu S, Liu H, Ma X, Han L, et al. T-cell-derived extracellular vesicles regulate B-cell IgG production via pyruvate kinase muscle isozyme 2. FASEB J. 2019;33:12780-99.

61. Lu J, Wu J, Xie F, Tian J, Tang X, Guo H, et al. CD4+ $\mathrm{T}$ cell-released extracellular vesicles potentiate the efficacy of the HBsAg vaccine by enhancing B cell responses. Adv Sci (Weinh). 2019;6:1802219.

62. Zhang F, Li R, Yang Y, Shi C, Shen Y, Lu C, et al. Specific decrease in B-cell-derived extracellular vesicles enhances post-chemotherapeutic CD8 ${ }^{+} \mathrm{T}$ cell responses. Immunity. 2019;50:738-50.e7.

63. Pathria P, Louis TL, Varner JA. Targeting tumor-associated macrophages in cancer. 2019;40:310-27.

64. Tang PMK, Nikolic-Paterson DJ, Lan HY. Macrophages: versatile players in renal inflammation and fibrosis. Nat Rev Nephrol. 2019;15:144-58.

65. Liu R, An Y, Jia W, Wang Y, Wu Y, Zhen Y, et al. Macrophage-mimic shape changeable nanomedicine retained in tumor for multimodal therapy of breast cancer. J Control Release. 2020;321:589-601.

66. Hu C, Lei T, Wang Y, Cao J, Yang X, Qin L, et al. Phagocyte-membrane-coated and laser-responsive nanoparticles control primary and metastatic cancer by inducing anti-tumor immunity. Biomaterials. 2020;255:120159.

67. Zhou J, Li X, Wu X, Zhang T, Zhu Q, Wang X, et al. Exosomes released from tumor-associated macrophages transfer miRNAs that induce a Treg/Th17 cell imbalance in epithelial ovarian cancer. Cancer Immunol Res. 2018;6:1578-92.

68. Rayamajhi S, Nguyen TDT, Marasini R, Aryal S. Macrophage-derived exosome-mimetic hybrid vesicles for tumor targeted drug delivery. Acta Biomater. 2019;94:482-94.

69. Fang RH, Kroll AV, Zhang LJ. Nanoparticle-based manipulation of antigen-presenting cells for cancer immunotherapy. Small. 2015;11:5483-96.

70. Wculek SK, Cueto FJ, Mujal AM, Melero I, Krummel MF, Sancho D. Dendritic cells in cancer immunology and immunotherapy. Nat Rev Immunol. 2020;20:7-24.

71. Besse B, Charrier M, Lapierre V, Dansin E, Lantz O, Planchard D, et al. Dendritic cell-derived exosomes as maintenance immunotherapy after first line chemotherapy in NSCLC. Oncoimmunology. 2016;5:e1071008.

72. Kowal J, Arras G, Colombo M, Jouve M, Morath JP, Primdal-Bengtson B, et al. Proteomic comparison defines novel markers to characterize heterogeneous populations of extracellular vesicle subtypes. Proc Natl Acad Sci U S A. 2016;113:E968-77.

73. Lindenbergh MFS, Koerhuis DGJ, Borg EGF, van't Veld EM, Driedonks TAP, Wubbolts R, et al. Bystander T-cells support clonal T-cell activation by controlling the release of dendritic cell-derived immunestimulatory extracellular vesicles. Front Immunol. 2019;10:448.

74. Tkach M, Kowal J, Zucchetti AE, Enserink L, Jouve M, Lankar D, et al. Qualitative differences in T-cell activation by dendritic cell-derived extracellular vesicle subtypes. EMBO J. 2017;36:3012-28.

75. Larssen P, Veerman RE, Akpinar GG, Hiltbrunner S, Karlsson MCI, Gabrielsson S. Allogenicity boosts extracellular vesicle-Induced antigen-specific immunity and mediates tumor protection and long-term memory in vivo. J Immunol. 2019;203:825-34.

76. Coffelt SB, Wellenstein MD, de Visser KE. Neutrophils in cancer: neutral no more. Nat Rev Cancer. 2016;16:431-46. 
77. Hernández JCC, Bracko O, Kersbergen CJ, Muse V, Haft-Javaherian M, Berg M, et al. Neutrophil adhesion in brain capillaries reduces cortical blood flow and impairs memory function in Alzheimer's disease mouse models. Nat Neurosci. 2019;22:413-20

78. Kolaczkowska E, Kubes P. Neutrophil recruitment and function in health and inflammation. Nat Rev Immunol. 2013;13:159-75.

79. Xue J, Zhao Z, Zhang L, Xue L, Shen S, Wen Y, et al. Neutrophil-mediated anticancer drug delivery for suppression of postoperative malignant glioma recurrence. Nat Nanotechnol. 2017;12:692-700.

80. Gao J, Wang S, Wang Z. High yield, scalable and remotely drug-loaded neutrophil-derived extracellular vesicles (EVs) for anti-inflammation therapy. Biomaterials. 2017;135:62-73.

81. Wu J, Lanier LL. Natural killer cells and cancer. Cancer Res. 2003;90:127-56.

82. Biron CA, Nguyen KB, Pien GC, Cousens LP, Salazar-Mather TP. Natural killer cells in antiviral defense: function and regulation by innate cytokines. Annu Rev Immunol. 1999;17:189-220.

83. Hayakawa Y, Smyth MJ. Innate immune recognition and suppression of tumors. 2006;95:293-322.

84. López-Soto A, Gonzalez S, Smyth MJ, Galluzzi LJCc. Control of metastasis by NK cells. Adv Cancer Res. 2017;32:135-54.

85. Sconocchia G, Arriga R, Tornillo L, Terracciano L, Ferrone S, Spagnoli GC. Melanoma cells inhibit NK cell functions. Cancer Res. 2012;72:5428-9.

86. Erdag G, Schaefer JT, Smolkin ME, Deacon DH, Shea SM, Dengel LT, et al. Immunotype and immunohistologic characteristics of tumor-infiltrating immune cells are associated with clinical outcome in metastatic melanoma. Cancer Res. 2012;72:1070-80.

87. Lorenzo-Herrero S, Lopez-Soto A, Sordo-Bahamonde C, Gonzalez-Rodriguez AP, Vitale M, Gonzalez S. NK Cell-Based Immunotherapy in cancer metastasis. Cancers (Basel). 2018;11:29.

88. Vivier E, Tomasello E, Baratin M, Walzer T, Ugolini S. Functions of natural killer cells. Nat Immunol. 2008;9:503-10.

89. Brandstadter JD, Yang Y. Natural killer cell responses to viral infection. J Innate Immun. 2011;3:274-9.

90. Ljunggren HG, Malmberg KJ. Prospects for the use of NK cells in immunotherapy of human cancer. Nat Rev Immunol. 2007;7:329-39.

91. Farcas M, Inngjerdingen M. Natural killer cell-derived extracellular vesicles in cancer therapy. Scand J Immunol. 2020;92:e12938.

92. Zhu L, Kalimuthu S, Oh JM, Gangadaran P, Baek SH, Jeong SY, et al. Enhancement of antitumor potency of extracellular vesicles derived from natural killer cells by IL-15 priming. Biomaterials. 2019;190191:38-50.

93. Wang G, Hu W, Chen H, Shou X, Ye T, Xu Y. Cocktail strategy based on NK cell-derived exosomes and their biomimetic nanoparticles for dual tumor therapy. Cancers (Basel). 2019;1:1560.

94. Zhang D, Zheng Y, Lin Z, Lan S, Zhang X, Zheng A, et al. Artificial engineered natural killer cells combined with antiheat endurance as a powerful strategy for enhancing photothermal-immunotherapy efficiency of solid tumors. Small. 2019;15:e1902636.

95. Hu CM, Fang RH, Wang KC, Luk BT, Thamphiwatana S, Dehaini D, et al. Nanoparticle biointerfacing by platelet membrane cloaking. Nature. 2015;526:118-21.

96. Han X, Chen J, Chu J, Liang C, Ma Q, Fan Q et al. Platelets as platforms for inhibition of tumor recurrence post-physical therapy by delivery of anti-PD-L1 checkpoint antibody. J Control Release. 2019;304:233-41.

97. Zhang X, Wang J, Chen Z, Hu Q, Wang C, Yan J, et al. Engineering PD-1-presenting platelets for cancer immunotherapy. Nano Lett. 2018;18:5716-25.

98. Wang C, Sun W, Ye Y, Hu Q Bomba HN, Gu Z. In situ activation of platelets with checkpoint inhibitors for post-surgical cancer immunotherapy. Nature Biomedical Engineering. 2017;1:0011. 
99. Hass R, Kasper C, Böhm S, Jacobs RJCC, Signaling. Different populations and sources of human mesenchymal stem cells (MSC): a comparison of adult and neonatal tissue-derived MSC. Cell Commun Signal. 2011;9:12.

100. Almalki SG, Agrawal DK. Key transcription factors in the differentiation of mesenchymal stem cells. Differentiation. 2016;92:41-51.

101. Jorgensen C, Djouad F, Apparailly F, Noel D. Engineering mesenchymal stem cells for immunotherapy. Gene Ther. 2003;10:928-31.

102. Lu JH, Peng BY, Chang CC, Dubey N, Lo WC, Cheng HC, et al. Tumor-targeted immunotherapy by using primary adipose-derived stem cells and an antigen-specific protein vaccine. Cancers (Basel). 2018;10:446.

103. Almeida-Porada G, Atala AJ, Porada CD. Therapeutic mesenchymal stromal cells for immunotherapy and for gene and drug delivery. Mol Ther Methods Clin Dev. 2020;16:204-24.

104. Chen D, Tang P, Liu L, Wang F, Xing H, Sun L, et al. Bone marrow-derived mesenchymal stem cells promote cell proliferation of multiple myeloma through inhibiting $\mathrm{T}$ cell immune responses via PD-1/PD-L1 pathway. Cell Cycle. 2018;17:858-67.

105. Najar M, Bouhtit F, Melki R, Afif H, Hamal A, Fahmi H, et al. Mesenchymal stromal cell-based therapy: new perspectives and challenges. J Clin Med. 2019;8:626.

106. Rani S, Ryan AE, Griffin MD, Ritter T. Mesenchymal stem cell-derived extracellular vesicles: toward cellfree therapeutic applications. Mol Ther. 2015;23:812-23.

107. Maude SL, Frey N, Shaw PA, Aplenc R, Barrett DM, Bunin NJ, et al. Chimeric antigen receptor T cells for sustained remissions in leukemia. N Engl J Med. 2014;371:1507-17.

108. Lai P, Weng J, Guo L, Chen X, Du X. Novel insights into MSC-EVs therapy for immune diseases. Biomark Res. 2019;7:6.

109. Stephen J, Bravo EL, Colligan D, Fraser AR, Petrik J, Campbell JD. Mesenchymal stromal cells as multifunctional cellular therapeutics - a potential role for extracellular vesicles. Transfus Apher Sci. 2016;55:62-9.

110. Del Fattore A, Luciano R, Pascucci L, Goffredo BM, Giorda E, Scapaticci M, et al. Immunoregulatory effects of mesenchymal stem cell-derived extracellular vesicles on T lymphocytes. Cell Transplant. 2015;24:2615-27.

111. Mardpour S, Hamidieh AA, Taleahmad S, Sharifzad F, Taghikhani A, Baharvand H. Interaction between mesenchymal stromal cell-derived extracellular vesicles and immune cells by distinct protein content. J Cell Physiol. 2019;234:8249-58.

112. Bruno S, Collino F, Iavello A, Camussi G. Effects of mesenchymal stromal cell-derived extracellular vesicles on tumor growth. Front Immunol. 2014;5:382.

113. Pascucci L, Cocce V, Bonomi A, Ami D, Ceccarelli P, Ciusani E, et al. Paclitaxel is incorporated by mesenchymal stromal cells and released in exosomes that inhibit in vitro tumor growth: a new approach for drug delivery. J Control Release. 2014;192:262-70.

114. Zhang K, Meng X, Yang Z, Cao Y, Cheng Y, Wang D, et al. Cancer cell membrane camouflaged nanoprobe for catalytic ratiometric photoacoustic imaging of microRNA in living mice. Adv Mater. 2019;31:e1807888.

115. JiangY, Krishnan N, Zhou J, ChekuriS, WeiX, Kroll AV, etal. Engineered cell-membrane-coated nanoparticles directly present tumor antigens to promote anticancer immunity. Adv Mater. 2020;32:e2001808.

116. Zhen X, Cheng P, Pu K. Recent sdvances in cell membrane-camouflaged nanoparticles for cancer phototherapy. Small. 2019;15:e1804105.

117. Lu Y, Dai J, Kong N, Liu J, Gong J, Yao Y. Internalization characterization of si nanorod with camouflaged cell membrane proteins reveals ATXN2 as a negative regulator. Cells. 2019;8:931. 
118. Zhang L, Zhang X, Lu G, Li F, Bao W, Song C, et al. Cell membrane camouflaged hydrophobic drug nanoflake sandwiched with photosensitizer for orchestration of chemo-photothermal combination therapy. Small. 2019;15:e1902648.

119. Yamada N, Kuranaga Y, Kumazaki M, Shinohara H, Taniguchi K, Akao Y. Colorectal cancer cell-derived extracellular vesicles induce phenotypic alteration of T cells into tumor-growth supporting cells with transforming growth factor- $\beta 1$ mediated suppression. Oncotarget. 2016;7:27033-43.

120. Zhao J, Schlosser HA, Wang Z, Qin J, Li J, Popp F, et al. Tumor-derived extracellular vesicles inhibit natural killer cell function in pancreatic cancer. Cancers (Basel). 2019;11:874.

121. Fleming V, Hu X, Weller C, Weber R, Groth C, Riester Z, et al. Me8lanoma extracellular vesicles generate immunosuppressive myeloid cells by upregulating PD-L1 via TLR4 signaling. Cancer Res. 2019;79:4715-28.

122. Horrevorts SK, Stolk DA, van de Ven R, Hulst M, van Het Hof B, Duinkerken S, et al. Glycan-modified apoptotic melanoma-derived extracellular vesicles as antigen source for anti-tumor vaccination. Cancers (Basel). 2019;11:1266.

123. Felgner S, Kocijancic D, Frahm M, Weiss S. Bacteria in cancer therapy: renaissance of an old concept. Int J Microbiol. 2016;2016:8451728.

124. Zhou S, Gravekamp C, Bermudes D, Liu K. Tumour-targeting bacteria engineered to fight cancer. Nat Rev Cancer. 2018;18:727-43.

125. Zhao M, Yang M, Li XM, Jiang P, Baranov E, Li S, Xu M, et al. Tumor-targeting bacterial therapy with amino acid auxotrophs of GFP-expressing Salmonella typhimurium. 2005;102:755-60.

126. Leventhal DS, Sokolovska A, Li N, Plescia C, KolodziejSA, Gallant CW, et al. Immunotherapy with engineered bacteria by targeting the STING pathway for anti-tumor immunity. Nat Commun. 2020;11:2739.

127. Gurbatri CR, Lia I, Vincent R, Coker C, Castro S, Treuting PM et al. Engineered probiotics forlocal tumor delivery of checkpoint blockade nanobodies. Sci Transl Med. 2020;12:eaax0876.

128. Liu L, Wu J, Gao J, Lu X. Bacteria-derived nanoparticles: multifunctional containers for diagnostic and therapeutic applications. Adv Healthc Mater. 2020;9:e2000893.

129. Yan S, Luo Z, Li Z, Wang Y, Tao J, Gong C, et al. Improving cancer immunotherapy outcomes using biomaterials. Angew Chem Int Ed Engl. 2020;[Epub ahead of print].

130. Chowdhury S, Castro S, Coker C, Hinchliffe TE, Arpaia N, Danino T. Programmable bacteria induce durable tumor regression and systemic antitumor immunity. Nat Med. 2019;25:1057-63.

131. Sedighi M, Zahedi Bialvaei A, Hamblin MR, Ohadi E, Asadi A, Halajzadeh M, et al. Therapeutic bacteria to combat cancer; current advances, challenges, and opportunities. Cancer Med. 2019;8:3167-81.

132. Yi X, Zhou H, Chao Y, Xiong S, Zhong J, Chai Z, et al. Bacteria-triggered tumor-specific thrombosis to enable potent photothermal immunotherapy of cancer. Sci Adv. 2020;6:eaba3546.

133. Xu C, Zhu W, Mao H, Zhang W, Yin GQ, Zhang XE, et al. Switch from polymorphic to homogenous selfassembly of virus-like particles of simian virus 40 through double-cysteine substitution. Small. 2020;16:e2004484.

134. Chen CY, Hutzen B, Wedekind MF, Cripe TP. Oncolytic virus and PD-1/PD-L1 blockade combination therapy. Oncolytic Virother. 2018;7:65-77.

135. LaRocca CJ, Warner SG. Oncolytic viruses and checkpoint inhibitors: combination therapy in clinical trials. Clin Transl Med. 2018;7:35.

136. Kerstetter-Fogle A, Shukla S, Wang C, Beiss V, Harris PLR, Sloan AE, et al. Plant virus-like particle in situ vaccine for intracranial glioma immunotherapy. Cancers (Basel). 2019;11:515.

137. Zhang P, Chen Y, Zeng Y, Shen C, Li R, Guo Z, et al. Virus-mimetic nanovesicles as a versatile antigendelivery system. Proc Natl Acad Sci U S A. 2015;112:E6129-38. 
138. Sayour EJ, McLendon P, McLendon R, De Leon G, Reynolds R, Kresak J, et al. Increased proportion of FoxP3 ${ }^{+}$regulatory $\mathrm{T}$ cells in tumor infiltrating lymphocytes is associated with tumor recurrence and reduced survival in patients with glioblastoma. Cancer Immunol Immunother. 2015;64:419-27.

139. Saha D, Martuza RL, Rabkin SD. Macrophage polarization contributes to glioblastoma eradication by combination immunovirotherapy and immune checkpoint blockade. Cancer Cell. 2017;32:253-67.e5.

140. Jiang H, Rivera-Molina Y, Gomez-Manzano C, Clise-Dwyer K, Bover L, Vence LM, et al. Oncolytic adenovirus and tumor-targeting immune modulatory therapy improve autologous cancer vaccination. Cancer Res. 2017;77:3894-907.

141. Lebel ME, Chartrand K, Tarrab E, Savard P, Leclerc D, Lamarre A. Potentiating cancer immunotherapy using papaya mosaic virus-derived nanoparticles. Nano Lett. 2016;16:1826-32.

142. Martikainen M, Essand M. Virus-based immunotherapy of glioblastoma. Cancers (Basel). 2019;11:186.

143. Parodi A, Molinaro R, Sushnitha M, Evangelopoulos M, Martinez JO, Arrighetti N, et al. Bio-inspired engineering of cell- and virus-like nanoparticles for drug delivery. Biomaterials. 2017;147:155-68.

144. Jin JW, Tang SQ Rong MZ, Zhang MQ. Synergistic effect of dual targeting vaccine adjuvant with aminated $\beta$-glucan and CpG-oligodeoxynucleotides for both humoral and cellular immune responses. Acta Biomater. 2018;78:211-23.

145. Tsoni SV, Brown GD. beta-Glucans and dectin-1. Ann N Y Acad Sci. 2008;1143:45-60.

146. Sahasrabudhe NM, Tian L, van den Berg M, Bruggeman G, Bruininx E, Schols HA, et al. Endo-glucanase digestion of oat $\beta$-Glucan enhances Dectin-1 activation in human dendritic cells. J Funct Foods. 2016;21:104-12.

147. Zhang M, Chun L, Sandoval V, Graor H, Myers J, Nthale J, et al. Systemic administration of $\beta$-glucan of $200 \mathrm{kDa}$ modulates melanoma microenvironment and suppresses metastatic cancer. Oncoimmunology. 2018;7:e1387347.

148. Yang Z, Xu M, Jia Z, Zhang Y, Wang L, Zhang H, et al. A novel antigen delivery system induces strong humoral and CTL immune responses. Biomaterials. 2017;134:51-63.

149. Liu H, Jia Z, Yang C, Song M, Jing Z, Zhao Y, et al. Aluminum hydroxide colloid vaccine encapsulated in yeast shells with enhanced humoral and cellular immune responses. Biomaterials. 2018;167:32-43.

150. Wiklander OP, Brennan MÁ, Lötvall J, Breakefield XO, Andaloussi SE. Advances in therapeutic applications of extracellular vesicles. Sci Transl Med. 2019;11:eaav8521.

151. Bosch M, Sanchez-Alvarez M, Fajardo A, Kapetanovic R, Steiner B, Dutra F, et al. Mammalian lipid droplets are innate immune hubs integrating cell metabolism and host defense. Science. 2020;370:eaay8085.

152. Zhang M, Viennois E, Xu C, Merlin D. Plant derived edible nanoparticles as a new therapeutic approach against diseases. Tissue Barriers. 2016;4:e1134415.

153. Yang C, Zhang M, Merlin D. Advances in plant-derived edible nanoparticle-based lipid nano-drug delivery systems as therapeutic nanomedicines. J Mater Chem B. 2018;6:1312-21.

154. Zhuang X, Deng ZB, Mu J, Zhang L, Yan J, Miller D, et al. Ginger-derived nanoparticles protect against alcohol-induced liver damage. J Extracell Vesicles. 2015;4:28713.

155. Veerman RE, Gucluler Akpinar G, Eldh M, Gabrielsson S. Immune cell-derived extracellular vesiclesfunctions and therapeutic applications. Trends Mol Med. 2019;25:382-94.

156. Banz A, Cremel M, Rembert A, Godfrin Y. In situ targeting of dendritic cells by antigen-loaded red blood cells: a novel approach to cancer immunotherapy. Vaccine. 2010;28:2965-72. 